\title{
Habilidades metacognitivas en adultos con abuso de sustancias bajo tratamiento en comunidad terapéutica
}

\section{Metacognitive abilities in adults with substance abuse treated in therapeutic community}

\author{
Felix Inchausti*, Javier Ortuño-Sierra**, Nancy V. García-Poveda***, Alejandro \\ BALLESTEROS-PRADOS****. \\ *Centro de Salud Mental de San Juan, Servicio Navarro de Salud-Osasunbidea, Pamplona-Iruña, España; **Departamento \\ de Psicología, Universidad Loyola Andalucía, Sevilla, España; ***Hospital San Juan de Dios, Santurce-Santurtzi, España; \\ ****Centro de Salud Mental de Estella, Servicio Navarro de Salud-Osasunbidea, Estella-Lizarra, España.
}

\section{Resumen}

Antecedentes: El término de metacognición hace referencia al conjunto de procesos psicológicos que permiten a los individuos desarrollar e integrar representaciones sobre los estados mentales propios y de los demás. El objetivo principal de este estudio fue examinar si los pacientes bajo tratamiento por consumo de sustancias, en régimen de comunidad terapéutica, presentan un perfil específico de déficits metacognitivos en las áreas de Autorreflexividad, Diferenciación, Descentramiento y Dominio, comparando sus puntuaciones con las obtenidas por dos muestras clínicas de pacientes con trastornos del espectro esquizofrénico y trastornos de ansiedad.

Método: Se diseñó un estudio con metodología mixta (cualitativacuantitativa). Se seleccionaron un total de 216 participantes con diagnósticos principales por consumo de sustancias $(n=52)$, espectro esquizofrénico $(n=49)$ y trastornos de ansiedad $(n=115)$. Los datos cualitativos se obtuvieron con la Entrevista de Evaluación de la Metacognición (MAI) y, posteriormente, estos fueron cuantificados con la Escala Abreviada de Evaluación de la Metacognición (MAS-A). Resultados: Las puntuaciones totales en la MAS-A del grupo con trastornos de ansiedad fueron estadísticamente superiores a las del grupo con trastornos por consumo de sustancias (TCS), y éstas, a su vez, fueron significativamente superiores a las del grupo con trastornos del espectro esquizofrénico. Por subescalas de la MAS-A, sólo hubo diferencias estadísticamente significativas entre las puntuaciones de Dominio del grupo con ansiedad y TCS, obteniendo el grupo con TCS puntuaciones estadísticamente equivalentes a las del grupo con trastornos del espectro esquizofrénico.

Conclusiones: De acuerdo con estos resultados, los programas actuales de intervención en drogadicción deberían orientarse más específicamente a mejorar las habilidades metacognitivas de Dominio. Palabras clave: Drogadicción; Metacognición; Escala Abreviada de Evaluación de la Metacognición (MAS-A); Rehabilitación, metodología mixta.

\begin{abstract}
Background: The term metacognition reflects a spectrum of psychological activities that allows people to form and integrate representations about their own mental states and those of others. The main goal of this study was to examine whether people with substance abuse disorders (SUDs), and treated in therapeutic community regime, displayed specific patterns of metacognitive deficits on Selfreflectivity, Understanding others' mind, Decentration, and Mastery, comparing their scores with two clinical groups of patients with schizophrenia spectrum disorders (SSDs) and anxiety disorders. Method:A mixed-methods (qualitative-quantitative) study was designed. Two hundred and sixteen adults aged 18-65 with principal diagnoses of SUDs $(n=52)$, SSDs $(n=49)$, and anxiety disorders $(n=115)$ were recruited. Qualitative data were obtained with the Metacognition Assessment Interview, which was then rated using a quantitative scale, the Metacognition Assessment Scale-Abbreviated (MAS-A).

Results: The anxiety disorders group had significantly higher MAS-A total scores than the SUDs group, and the SUDs group obtained significantly higher MAS-A total scores than the SSDs group. Concerning the MAS-A subscale scores, the SUDs group displayed significantly lower scores only on the Mastery subscale compared to the anxiety disorders group, with the SUDs and SSDs groups obtaining equivalent Mastery scores.

Conclusions: According to these findings, current interventions for addiction should focus more specifically on improving metacognitive Mastery.

Keywords: Addiction; Metacognition; Metacognition Assessment Scale-Abbreviated (MAS-A); Rehabilitation; mixed-methods.
\end{abstract}


$\mathrm{O}$ riginalmente, el término de metacognición fue utilizado en la literatura educativa para hacer referencia a la capacidad para reflexionar sobre el propio pensamiento cuando aprendemos (Flavell, 1979). Posteriormente, el uso de este concepto se ha extendido a numerosos campos de investigación, tales como el apego, la psicopatología, el desarrollo humano o la psicología cognitiva (Bacon y Izaute, 2009; Dinsmore et al, 2008; Tarricone, 2011). De forma amplia, este constructo alude tanto a los procesos mentales simples encargados, por ejemplo, de identificar los propios deseos, pensamientos o emociones, como los procesos complejos que nos permiten integrar la información intersubjetiva para crear representaciones amplias sobre uno mismo, los demás y el mundo (Semerari et al., 2003). Aunque este conjunto de habilidades han recibido múltiples denominaciones en la literatura (p.ej., cognición social, teoría de la mente, inteligencia emocional o mentalización), todas ellas apuntan a procesos mentales que subyacen a la experiencia interpersonal. En un intento por unificar el campo, Lysaker et al. (2005) han propuesto una definición general de metacognición que incluye cuatro grandes habilidades: (1) la Autorreflexividad o capacidad para pensar sobre los propios estados mentales; (2) la Diferenciación o capacidad para pensar sobre los estados mentales ajenos; (3) el Descentramiento o capacidad para comprender que uno no es el centro del mundo y que existen formas distintas de entender la realidad; y (4) el Dominio o capacidad para integrar la información intersubjetiva en definiciones amplias de los problemas que permitan responder de manera adaptativa.

A pesar de que existen numerosas pruebas para evaluar las habilidades metacognitivas de orden inferior (p.ej., con tareas para medir la capacidad de reconocer y expresar emociones específicas; Caletti et al., 2013), aún no se dispone de tantos instrumentos de medida para valorar los procesos de orden superior que implican atender a cómo los individuos integran y responden en situaciones interpersonales de alto contenido emocional. Con objeto de superar esta limitación, recientemente se ha desarrollado la Entrevista de Evaluación de la Metacognición (Metacognition Assessment Interview, MAI; Semerari et al., 2012). La MAI es una entrevista semiestructurada que valora la actividad metacognitiva de los individuos ante episodios autobiográficos relevantes de tipo intersubjetivo. En concreto, la MAI solicita a los entrevistados que describan detalladamente el peor acontecimiento psicológico experimentado en los últimos meses. El objetivo principal es provocar narraciones vividas que permitan identificar todos los procesos metacognitivos puestos en marcha por el sujeto en esa situación. Una vez obtenida la narración, la información de las respuestas se ordena y puntúa

Tabla 1. Estructura de la Escala Abreviada de Evaluación de la Metacognición (MAS-A; Lysaker et al., 2005).

\begin{tabular}{|c|c|c|c|c|}
\hline Nivel & Autorreflexividad (S) & Diferenciación (0) & Descentramiento (D) & Dominio (M) \\
\hline 0 & $\begin{array}{l}\text { Ausencia total de conciencia sobre } \\
\text { la propia actividad mental }\end{array}$ & $\begin{array}{l}\text { Ausencia total de conciencia de la } \\
\text { actividad mental de otros }\end{array}$ & $\begin{array}{l}\text { Considerar que uno es el centro de } \\
\text { todo lo que ocurre }\end{array}$ & $\begin{array}{l}\text { Ausencia de conciencia de los } \\
\text { problemas }\end{array}$ \\
\hline 2 & $\begin{array}{l}\text { Conciencia de que los } \\
\text { pensamientos son propios }\end{array}$ & $\begin{array}{l}\text { Conciencia de que los otros tienen } \\
\text { actividad mental propia }\end{array}$ & $\begin{array}{l}\text { Conciencia de que existen formas } \\
\text { diferentes de entender un mismo } \\
\text { suceso }\end{array}$ & $\begin{array}{l}\text { Conciencia de los problemas como } \\
\text { resolubles pero con ausencia de } \\
\text { respuesta }\end{array}$ \\
\hline 3 & $\begin{array}{l}\text { Distinción de diferentes } \\
\text { operaciones cognitivas propias } \\
\text { (pensamientos, fantasías, } \\
\text { recuerdos...) }\end{array}$ & $\begin{array}{l}\text { Distinción de diferentes } \\
\text { operaciones cognitivas en los } \\
\text { otros (pensamientos, fantasías, } \\
\text { recuerdos...) }\end{array}$ & $\begin{array}{l}\text { Conciencia de que los hechos son } \\
\text { el resultado de factores múltiples } \\
\text { y complejos }\end{array}$ & Respuestas pasivas \\
\hline 4 & $\begin{array}{l}\text { Distinción de diferentes estados } \\
\text { emocionales }\end{array}$ & $\begin{array}{l}\text { Reconocimiento de diferentes } \\
\text { estados emocionales en los otros }\end{array}$ & - & Respuestas de búsqueda de ayuda \\
\hline 6 & $\begin{array}{l}\text { Reconocimiento de que el deseo } \\
\text { no es la realidad }\end{array}$ & $\begin{array}{l}\text { Descripciones completas del } \\
\text { pensamiento de los otros a lo } \\
\text { largo del tiempo }\end{array}$ & - & Respuestas con cambios \\
\hline 7 & $\begin{array}{l}\text { Integración de los propios } \\
\text { pensamientos y emociones en una } \\
\text { narración }\end{array}$ & $\begin{array}{l}\text { Descripciones completas del } \\
\text { pensamiento de los otros a lo } \\
\text { largo de sus vidas }\end{array}$ & - & $\begin{array}{l}\text { Respuestas basadas en los } \\
\text { propios conocimientos }\end{array}$ \\
\hline 8 & $\begin{array}{l}\text { Integración de varias narraciones } \\
\text { reconociendo patrones a lo largo } \\
\text { del tiempo }\end{array}$ & - & - & $\begin{array}{l}\text { Respuestas basadas en el } \\
\text { conocimiento de los otros }\end{array}$ \\
\hline 9 & $\begin{array}{l}\text { Reconocimiento de pensamientos } \\
\text { y emociones conectadas a través } \\
\text { de su propia vida }\end{array}$ & - & - & $\begin{array}{l}\text { Respuestas basadas en una } \\
\text { comprensión amplia de la vida }\end{array}$ \\
\hline
\end{tabular}


con la Escala Abreviada de Evaluación de la Metacognición (Metacognition Assessment Scale-Abbreviated, MAS-A; Lysaker et al., 2005). La MAS-A es una escala breve que fue desarrollada específicamente para analizar datos de tipo cualitativo. Como se puede observar en la Tabla 1, la MAS-A consta de cuatro subescalas que miden las cuatro habilidades metacognitivas descritas anteriormente. Puntuaciones altas indican una mayor capacidad para crear representaciones amplias sobre uno mismo, los demás y el mundo, así como para utilizar estas representaciones para responder adecuadamente a desafíos de tipo psicológico y social (Lysaker et al., 2005). Estudios recientes han encontrado que las puntuaciones de la MAS-A correlacionan significativamente con variables clínicas como la gravedad de los síntomas o el nivel de funcionamiento en varios trastornos mentales, incluyendo la esquizofrenia (McLeod et al., 2014), los trastornos de personalidad (Semerari et al., 2014) o la depresión (Ladegaard, Lysaker, Larsen y Videbech, 2014).

En el caso concreto de los trastornos relacionados con el consumo de sustancias (TCS), se ha visto que la alexitimia, esto es, la dificultad para nombrar y expresar emociones propias, correlaciona significativamente con el abuso de sustancias (Thorberg, Young, Sullivan y Lyvers, 2009). En este sentido, Highland, Herschl, Klanecky y McChargue (2013) han descubierto que la expresión de determinados genes acentúa esta relación entre alexitimia y consumo de sustancias. Por otro lado, Lysaker et al. (2014) han encontrado que las habilidades metacognitivas relacionadas con el uso de los estados mentales para resolver problemas sociales (o capacidad de Dominio), moderan el efecto de la alexitimia sobre el consumo. Paralelamente, Wasmuth et al. (2015) corroboraron que las personas con problemas de consumo presentan déficits más marcados en las habilidades de Dominio frente a controles con VIH+ sin historia de consumos. En cuanto al tipo de sustancia, Roser et al. (2012) han asociado el consumo de cannabis con déficits significativos en la capacidad para inferir estados mentales en otros. Este estudio encontró, además, que el consumo crónico de cannabis provocaba patrones de activación neuronal muy similares al de las personas con riesgo a psicosis. En otro trabajo de Gizewski et al. (2013), se observó que el consumo abusivo de alcohol afectaba a las áreas cerebrales relacionadas con la empatía cognitiva y afectiva. Existen evidencias empíricas que muestran que los déficits en metacognición correlacionan con peores resultados en los tratamientos de deshabituación (Saladin et al., 2012; Thorberg et al., 2011), el tipo de sustancia consumida, la abstinencia y el riesgo de recaída (Toneatto, 1999), las autolesiones (Verrocchio, Conti y Fulcheri, 2010), el malestar emocional (de Rick, Vanheule y Verhaeghe, 2009) y el abuso de alcohol en muestras no clínicas (Lyvers, Onuoha, Thorberg y Samios, 2012).

En definitiva, los resultados de la literatura hasta la fecha parecen subrayar la relevancia y el interés de estudiar este conjunto de habilidades en personas con abuso de sustan- cias. El hecho de que, además, aún no esté claro si existen perfiles metacognitivos diferentes según el tipo de trastorno o cuáles son los efectos de los tratamientos actuales sobre la metacognición, requiere seguir investigando estas cuestiones de cara a mejorar la comprensión de los procesos psicológicos que subyacen a las drogadicciones y su tratamiento. En relación a los tipos de intervención, aunque existe evidencia de que las drogadicciones provocan cambios neuropatológicos importantes, la capacidad para actuar sobre ellos aún es bastante limitada (Bart, 2012). En este sentido, el nivel de análisis metacognitivo puede aportar nuevas opciones de tratamiento psicoterapéutico y/o ayudar a optimizar los ya existentes. Por ejemplo, se ha visto que las personas que puntúan bajo en Autorreflexividad responden mejor a intervenciones de tipo individual (Lysaker et al., 2013). Contrariamente, es de suponer que las personas con puntuaciones bajas en Descentramiento se beneficien más de intervenciones grupales que les permitan mejorar su comprensión de los estados mentales ajenos (Wasmuth et al., 2015). Puntuaciones bajas en todas las áreas de la metacognición justificarían el uso de ambos formatos de intervención, así como de otras de tipo interdisciplinar que actúen sobre todos los déficits y sus repercusiones funcionales.

Dentro de este contexto de investigación, el propósito de este estudio fue examinar las habilidades metacognitivas de un grupo de pacientes con TCS bajo tratamiento de deshabituación en régimen de comunidad terapéutica. Concretamente se pretendió determinar si los pacientes con TCS presentaban diferencias en las habilidades metacognitivas, evaluadas con la MAS-A, en comparación con pacientes con trastornos del espectro esquizofrénico y con trastornos de ansiedad leves. La selección de estos grupos de comparación estuvo motivada por las siguientes razones. Estudios recientes sugieren que el uso de controles sanos -esto es, individuos sin problemas específicos de salud- no son un buen grupo para comparar la capacidad metacognitiva de pacientes con problemas de salud mental, como es el caso del grupo con TCS (Wasmuth et al., 2015). Específicamente, este planteamiento arguye que el uso de controles sanos podría infravalorar su capacidad metacognitiva por el simple hecho de presentar un problema de salud (p.ej., Lysaker et al., 2012). Por este motivo, la inclusión del grupo de pacientes con trastornos de ansiedad leves pretendió garantizar que todos los participantes del estudio tuvieran algún problema de salud, y que, además, recibieran algún tipo de tratamiento de salud mental (psicoterapéutico y/o psicofarmacológico), aunque fuera de baja intensidad, en el momento de la evaluación. Los pacientes con trastornos del espectro esquizofrénico se incluyeron por ser el grupo con déficits metacognitivos más claramente establecidos en la literatura (véase p.ej., Bacon y Izaute, 2009), aportando datos relevantes sobre todo de los niveles inferiores del constructo. En línea con estos hallazgos, las hipótesis del estudio fueron las siguientes. En primer lugar, se esperó que el gru- 
po de pacientes con trastornos del espectro esquizofrénico obtuviera las puntuaciones más bajas en las subescalas de la MAS-A. En segundo lugar, se esperó que, partiendo del nivel superior de funcionamiento de los pacientes con trastornos leves de ansiedad frente a los pacientes con TCS, el grupo con ansiedad presentara puntuaciones superiores a las del grupo con TCS en la MAS-A.

\section{Método}

\section{Participantes}

El estudio incluyó tres grupos de pacientes. El primer grupo lo formaron un total de 52 adultos con TCS bajo proceso de deshabituación en régimen de comunidad terapéutica y clínicamente estabilizados (sin hospitalizaciones ni cambios de medicación en el último mes). Dentro de este grupo, 21 participantes presentaron un diagnostico principal de trastorno por consumo de varias sustancias, 13 por consumo de alcohol, 10 por consumo de opiáceos y 8 por consumo de cocaína.

Un segundo grupo incluyó a 49 adultos con trastornos del espectro esquizofrénico, en concreto: esquizofrenia ( $n$ $=33)$, trastorno esquizoafectivo $(n=15)$ y trastorno delirante $(n=1)$, según criterios CIE-10 (OMS, 1992), bajo tratamiento en régimen de hospital de día y clínicamente estabilizados (sin cambios en la medicación antipsicótica en los últimos 6 meses).

El tercer grupo lo formaron 115 adultos con trastornos de ansiedad leves pertenecientes a dos centros ambulatorios de salud mental y con diagnósticos principales de, al menos, un trastorno de ansiedad, según criterios CIE-10. Dentro de este tercer grupo, 42 participantes presentaron un diagnóstico principal de trastorno de pánico con agorafobia, $24 \mathrm{de}$ ansiedad generalizada, 19 de pánico sin agorafobia, 18 de fobia social y 12 de agorafobia sin pánico. Las características sociodemográficas de los participantes se presentan en la Tabla 2.

Todos los diagnósticos y valoraciones clínicas se hicieron por psiquiatras o psicólogos clínicos externos a la investigación. Los criterios generales de exclusión del estudio fueron: sospecha o diagnóstico de retraso mental o de trastornos generalizados del desarrollo, presencia de síndromes neurológicos (demencias, epilepsia, esclerosis múltiple, etc.), problemas sensoriales (ceguera, sordera, etc.) o dificultades de comprensión (p.ej., no hablar castellano) y no firmar o no tener capacidad para firmar el consentimiento informado. Se excluyó también a los participantes con síntomas psicóticos positivos severos o extremos [puntuaciones $\geq 4$ en cualquier ítem de la Escala de Evaluación de los Síntomas Positivos (SAPS; Adrenasen, 1984)] y los participantes con trastornos de ansiedad moderados o graves [puntuaciones totales $\geq 30$ en el Inventario de Ansiedad de Beck (BAI; Sanz y Navarro, 2003)]. Por último, la presencia o sospecha de TCS fue un criterio de exclusión en los grupos del espectro esquizofrénico y de ansiedad.

\section{Instrumentos}

Entrevista de Evaluación de la Metacognición (Metacognition Assessment Interview, MAI; Semerari et al., 2012). La MAI es una entrevista clínica semiestructurada de 30 a 60 minutos de duración. En el contexto de la entrevista, se solicita al individuo que relate la experiencia o el suceso más desagradable experimentado en los últimos 6 meses. Los requisitos son que el episodio sea de naturaleza autobiográfica, relevante a nivel personal y que incluya interacciones con otras personas. En un primer momento, las preguntas son intencionalmente abiertas para permitir la narración libre; esto permite que las habilidades metacognitivas puestas en marcha por el entrevistado en el suceso afloren espontáneamente. Posteriormente, se hacen preguntas concretas con el objetivo de valorar específicamente cada habilidad metacognitiva. El audio de todas las entrevistas se grabó para su posterior transcripción y cuantificación con la MAS-A.

Escala Abreviada de Evaluación de la Metacognición (Metacognition Assessment Scale-Abbreviated, MAS-A; Lysaker et al., 2005). La MAS-A es una escala que evalúa los cuatro tipos de actividad metacognitiva descritos anteriormente (Lysaker et al., 2005; Semerari et al., 2003). Este instrumento es una adaptación breve de la Escala de Evaluación de la

Tabla 2. Características sociodemográficas de los participantes.

\begin{tabular}{|c|c|c|c|c|c|c|}
\hline & $\begin{array}{l}\text { Grupo } 1 \text { Ansiedad } \\
(n=115)\end{array}$ & $\begin{array}{l}\text { Grupo } 2 \text { Drogadicción } \\
(n=52)\end{array}$ & $\begin{array}{l}\text { Grupo } 3 \text { Esquizofrenia } \\
(n=49)\end{array}$ & & & \\
\hline Variable & fo Media (DT) & fo Media (DT) & fo Media $(D T)$ & $F$ & $\eta^{2}$ parcial & Análisis Post hoc \\
\hline \multicolumn{7}{|l|}{ Sexo } \\
\hline Hombres & 32 & 41 & 26 & & & \\
\hline Mujeres & 83 & 11 & 23 & & & \\
\hline Edad & $43,07(15,54)$ & $36,86(9,18)$ & $37,69(12,62)$ & $4,94^{\star}$ & 0,07 & $1>2,3$ \\
\hline Años de educación & $13,42(1,97)$ & $12,64(1,92)$ & $12,44(2,73)$ & $4,57^{*}$ & 0,05 & $1>2,3$ \\
\hline
\end{tabular}

Nota. $f=$ frecuencia; $D T=$ desviación típica; $\eta^{2}$ parcial $=$ eta cuadrado parcial. ${ }^{\star} p<0,05$ 
Metacognición (Metacognition Assessment Scale, MAS; Semerari et al., 2003) que cuantifica las habilidades metacognitivas implícitas en las respuestas verbales de la entrevista. Consta de cuatro subescalas: 'Autorreflexividad' (Self-reflectivity), que incluye nueve niveles sobre la capacidad para pensar y formar ideas cada vez más plausibles e integradas de uno mismo; 'Diferenciación' (Mind of Others), que consta de siete niveles sobre la capacidad para pensar y formar ideas cada vez más complejas y plausibles sobre los demás; 'Descentramiento' (Decentration), compuesta por tres niveles que miden la capacidad para formar ideas integradas sobre uno mismo y los demás; y, por último, 'Dominio' (Mastery), que incluye nueve niveles sobre la capacidad para utilizar los estados mentales propios y ajenos para responder a problemas psicológicos y sociales (véase la tabla 1). A mayores puntuaciones en las subescalas, mayor capacidad para integrar y utilizar eficazmente la información intersubjetiva. Los datos obtenidos hasta la fecha con la versión norteamericana de la MAS-A sugieren unos valores de consistencia interna y fiabilidad test-retest e inter-jueces aceptables, con coeficientes intraclase entre 0,71 y 0,91 (Lysaker et al, 2005; Lysaker y Salyers, 2007). En cuanto a las evidencias de validez sobre el constructo teórico, las puntuaciones de la MAS-A correlacionan significativamente con otros test que miden conciencia de enfermedad, insight cognitivo, complejidad de los esquemas sociales o la preferencia por estrategias activas de afrontamiento en personas con psicosis (Lysaker et al., 2015).

Dado que ni la escala MAS-A ni la entrevista MAI están todavía validadas al castellano, en este estudio se han empleado versiones piloto de ambas pruebas (más información sobre ambas traducciones puede solicitarse al autor principal FI). La traducción y adaptación al castellano se llevó a cabo siguiendo las directrices de la Comisión Internacional de Tests (Muñiz, Elosua y Hambleton, 2013). La cuantificación con la MAS-A de los datos cualitativos obtenidos en la MAI se llevó a cabo por dos jueces externos a la investigación expertos en el uso de ambos instrumentos. Los jueces puntuaron a cada participante en función de las transcripciones de la MAI por lo que desconocieron en todo momento las hipótesis del estudio y las características de los participantes. La fiabilidad inter-jueces de las puntuaciones de la MAS-A fue de 0,91 .

\section{Procedimiento}

El protocolo del estudio fue aprobado por el comité ético institucional de los centros donde se recogieron los datos. Tras explicar a todos los participantes los objetivos, beneficios y posibles riesgos de la investigación, los que de manera voluntaria aceptaron participar debían de firmar un consentimiento informado previamente a la recogida de los datos. La participación en el estudio no supuso ningún tipo de contraprestación o incentivo. La información obtenida fue almacenada asegurando la total confidenciali- dad de los datos. La recogida de los datos se realizó en una única entrevista de 30 a 60 minutos de duración aproximada en el contexto de las sesiones generales de evaluación o seguimiento clínico de los casos. Todas las entrevistas fueron realizadas por dos psicólogos clínicos entrenados en el uso de la MAI.

\section{Análisis de datos}

Los análisis de datos se llevaron a cabo en dos fases utilizando el paquete estadístico SPSS versión 21 (IBM Corp., 2012). En un primer momento, las variables sociodemográficas edad y nivel educativo de los participantes de los tres grupos (TCS, espectro esquizofrénico y ansiedad) se compararon para determinar si era necesario emplear alguna de estas variables como covariable en los análisis posteriores. En un segundo momento, se llevaron a cabo los análisis de varianza (ANOVA) o, en su caso, de covarianza (ANCOVA) para comparar las puntuaciones medias totales de la MAS-A entre los grupos objeto de comparación. En el caso de que estas diferencias fueran estadísticamente significativas $(p<$ $0,05)$, se procedió a comparar las puntuaciones medias en las subescalas de la MAS-A. Como estimador de la magnitud de los efectos se utilizó el estadístico eta cuadrado parcial $\left(\eta^{2}\right.$ parcial $)$.

\section{Resultados}

La tabla 3 presenta el promedio de las puntuaciones totales y por subescalas obtenidas en la MAS-A por los participantes de los grupos TCS, espectro esquizofrénico y trastornos de ansiedad. Los ANOVA llevados a cabo señalaron que los grupos difirieron en edad y nivel educativo; en concreto, la edad y el nivel de estudios promedio del grupo con trastornos de ansiedad fueron superiores a los del grupo de trastornos del espectro esquizofrénico y TCS $(p<0,05)$. Para controlar el posible efecto de ambas variables en las posteriores comparaciones, la edad y el nivel educativo fueron incluidas como covariables.

Los participantes del grupo de ansiedad obtuvieron puntuaciones totales en la MAS-A significativamente más altas que los del grupo con TCS, y este grupo a su vez puntuó significativamente más alto que el grupo de trastornos del espectro esquizofrénico $(p<0,001)$. Por subescalas, los participantes del grupo con trastornos de ansiedad y TCS obtuvieron, tras controlar el efecto de las variables edad y nivel educativo, puntuaciones significativamente más altas en Autorreflexividad, Diferenciación y Descentramiento que el grupo con trastornos del espectro esquizofrénico. Los participantes del grupo con trastornos de ansiedad puntuaron significativamente más alto en la subescala de Dominio de la MAS-A en comparación con los otros dos grupos de participantes. Finalmente, se encontraron puntuaciones equivalentes en esta subescala en los grupos de TCS y del espectro esquizofrénico (véase la tabla 3). 
Tabla 3. Resultados de los ANCOVA, tamaños del efecto y análisis post hoc con las puntuaciones en metacognición, controlando la edad y el nivel educativo de los participantes.

\begin{tabular}{|c|c|c|c|c|c|c|}
\hline & $\begin{array}{l}\text { Grupo } 1 \text { Ansiedad } \\
(n=115)\end{array}$ & $\begin{array}{l}\text { Grupo } 2 \text { Drogadicción } \\
(n=52)\end{array}$ & $\begin{array}{l}\text { Grupo } 3 \text { Esquizofrenia } \\
(n=49)\end{array}$ & & & \\
\hline & Media $(D T)$ & Media $(D T)$ & Media $(D T)$ & $F$ & $\eta^{2}$ parcial & Análisis Post hoc \\
\hline \multicolumn{7}{|l|}{ MAS-A } \\
\hline Autorreflexividad & $6,05(1,61)$ & $6,00(1,66)$ & $4,08(1,19)$ & $32,74^{\star *}$ & 0,17 & $1,2>3$ \\
\hline Diferenciación & $4,00(1,26)$ & $3,91(1,01)$ & $2,69(0,98)$ & $29,81^{\star \star}$ & 0,15 & $1,2>3$ \\
\hline Descentramiento & $1,52(0,98)$ & $1,14(1,22)$ & $0,99(0,92)$ & $6,06^{\star \star}$ & 0,09 & $1,2>3$ \\
\hline Dominio & $4,70(1,71)$ & $3,51(1,34)$ & $3,36(1,56)$ & $18,24^{\star \star}$ & 0,14 & $1>2,3$ \\
\hline Total & $16,26(4,96)$ & $14,56(4,72)$ & $11,12(4,01)$ & $26,95^{\star \star}$ & 0,19 & $1>2>3$ \\
\hline
\end{tabular}

Nota. MAS-A = Escala Abreviada de Evaluación de la Metacognición; $D T=$ desviación típica; $\mathrm{n}^{2}$ parcial = eta cuadrado parcial. ** $p<0,001$.

\section{Discusión}

El objetivo principal de este estudio fue examinar las habilidades metacognitivas, evaluadas con la MAS-A, de un grupo de pacientes con TCS. Asimismo, se pretendió determinar si sus habilidades metacognitivas difieren de las observadas en otros pacientes con trastornos del espectro esquizofrénico y trastornos de ansiedad leves. Para tal fin, se seleccionaron un total de 216 adultos bajo tratamiento por TCS, trastornos del espectro esquizofrénico o trastornos de ansiedad. Todos fueron entrevistados en primer lugar con la MAI y, posteriormente, sus respuestas se cuantificaron por dos jueces ciegos utilizando la MAS-A. A la luz de los resultados obtenidos, parece que: (1) los pacientes con TCS demostraron rendimientos estadísticamente equivalentes en el área de Dominio al de los pacientes con trastornos del espectro esquizofrénico; y (2) los participantes con TCS no demostraron peores rendimientos metacognitivos en las áreas de Autorreflexividad, Diferenciación y Descentramiento que el de los pacientes con trastornos de ansiedad leves.

Estos hallazgos se alejan de la conceptualización de las drogadicciones como conductas de tipo impulsivo o compulsivo fuertemente asociadas con una pobre capacidad para la autorreflexión (Chambers y Potenza, 2003; O'Brien, 2008). Por ejemplo, muchos de los programas de deshabituación actuales, como el de Alcohólicos Anónimos (2001), parten de un modelo que caracteriza este tipo de trastornos muy vinculados con una falta de autocontrol y autopercepción de los estados mentales. Las altas prevalencias de alexitimia (Lysaker et al., 2014), la escasa flexibilidad cognitiva (Luoma, Drake, Kohlenberg y Hayes, 2011), las dificultades para las relaciones interpersonales (Greene et al., 1999) o el pobre autoconcepto (Chelton y Bonney, 1987) manifestados en los TCS apoyarían este tipo de definiciones de las drogadicciones. Sin embargo, los resultados de este estudio apuntan en la dirección de otros trabajos recientes que sugieren que el problema de las personas con TCS no estaría tanto en sus dificultades para describir y comprender los pensamientos, emociones o intenciones propios y de los demás, sino en su pobre capacidad para regular e integrar esta información y poner en marcha conductas adaptativas (Lysaker et al., 2014; Wasmuth et al., 2015).

Asimismo, los resultados de este estudio apoyan la idea de que el abuso de sustancias per se no altera las funciones metacognitivas (Wasmuth et al., 2015) y ofrecen una hipótesis alternativa para entender la etiología de los déficits funcionales frecuentemente asociados a los TCS. Esta hipótesis se basa en el hecho de que es posible que el consumo pueda explicarse como una conducta compensatoria de los déficits en las habilidades metacognitivas de Dominio (Wasmuth et al., 2015). El consumo de sustancias, al ser una actividad conocida, controlable y fácilmente manejable, no requiere de esfuerzos metacognitivos importantes como sí precisan, por el contrario, otras actividades sociales y ocupacionales. Puesto que los circuitos neurobiológicos subyacentes a las actividades gratificantes y al consumo son similares (Chambers, Bickel y Potenza, 2007), podría ser razonable suponer que las adicciones compensen las dificultades para conseguir recompensas en otros contextos más complejos. Sin embargo, no debe descartarse la hipótesis alternativa de que los déficits en las habilidades de Dominio sean una consecuencia parcial o total del consumo crónico de sustancias (Lysaker et al., 2014).

Por otro lado, las similitudes observadas en este estudio en la capacidad metacognitiva de Dominio entre los participantes con trastornos del espectro esquizofrénico y con TCS pueden llevar a sospechar de la presencia de mecanismos neuronales subyacentes comunes o, al menos, de ciertas similitudes en la forma de procesar la información en ambos trastornos. No obstante, esto también puede deberse a otras muchas causas, como los estilos de apego (Fonagy y Bateman, 2006), las variables genéticas (Highland et al., 2013), la motivación (Bachiller et al., 2015) o la vivencia de experiencias traumáticas (Pec, Bob y Lysaker, 2015). Futuros estudios deberán dirigirse a esclarecer si este tipo de déficits comparten mecanismos neuronales similares y/o si tienen un origen común. 
Como limitaciones principales de este estudio cabe enumerar las siguientes. En primer lugar, el tamaño reducido de los grupos, sobre todo en el espectro esquizofrénico y TCS, y la sobrerrepresentación de las mujeres en el grupo de ansiedad y de los varones en el grupo de TCS, afectan claramente a la validez y extrapolación de los resultados a otras muestras de interés. Además, puesto que las conductas de consumo están muy generalizadas y arraigadas en nuestra sociedad, como ocurre por ejemplo con el alcohol o el cannabis, estos problemas pasan a menudo desapercibidos y no son diagnosticados. El riesgo de que los datos estén contaminados por la presencia de participantes con TCS comórbidos en los grupos del espectro esquizofrénico y ansiedad, supone una limitación potencial a la validez de los resultados. En segundo lugar, el hecho de que las conclusiones se sustenten en las puntuaciones obtenidas con versiones piloto de la entrevista MAI y la escala MAS-A (ambas pruebas aún no validadas al castellano) supone una limitación que afecta a las evidencias de validez de las estimaciones del constructo "metacognición”. Asimismo, las dos técnicas de evaluación utilizadas se basan en informaciones verbales proporcionadas por los individuos, por lo que no es descartable que existan discrepancias entre las habilidades metacognitivas reales y las descritas en la entrevista. Futuros estudios deberán emplear otros métodos de evaluación convergente, tales como los basados en el análisis de la interacción social o las técnicas individuales no verbales donde sea posible inferir el uso de estrategias metacognitivas a partir de patrones repetidos de la conducta manifestada mientras se realizan determinadas tareas. El nivel de desarrollo actual en el campo de la metacognición parece señalar que ninguna técnica por sí sola es suficiente para valorar estos procesos y es necesario apuntar hacia el uso de varios marcadores metacognitivos. En tercer lugar, y desde un punto de vista práctico, aunque este estudio pone de manifiesto la relevancia de que futuras investigaciones examinen la eficacia de los tratamientos basados en la metacognición en TCS, como por ejemplo, el entrenamiento metacognitivo (van Oosterhout et al., 2015) o el de habilidades sociales centrado en la metacognición (Ottavi et al., 2014), este trabajo no aporta información detallada sobre las variables que podrían mejorar la metacognición o sobre los efectos que intervenciones dirigidas específicamente a estos déficits pueden tener en poblaciones clínicas concretas. Hay que destacar también que si bien el consumo podría ser una conducta compensatoria de los déficits metacognitivos, es muy posible que estos se manifiesten de manera exagerada como consecuencia de los cambios neuropatológicos asociados al consumo crónico de drogas (Chambers, 2013; Volkow, Fowler, Wang, Baler y Telang, 2009). Finalmente, aunque la selección de los grupos se realizó con objeto de atenuar los posibles efectos derivados de padecer un problema de salud (mental) y del tratamiento psicológico y/o psicofarmacológico recibido, ambas variables no fueron controladas de manera específica (diagnóstico específico, tipo y duración del tratamiento, fármaco, etc.), así como tampoco su potencial impacto sobre la capacidad metacognitiva de los participantes (López-Duran et al., 2006).

Futuras líneas de investigación deberán dirigirse a explorar con más detenimiento las relaciones que existen entre las conductas adictivas, las habilidades metacognitivas, el nivel de funcionamiento personal, ocupacional y social, así como las bases neurobiológicas implicadas. Parece relevante, además, analizar con mayor énfasis el papel que pueden jugar las variables metacognitivas en general y las de Dominio en particular a la hora de predecir el mantenimiento de la abstinencia. En esta dirección, cabe suponer que las mejoras en las habilidades de Domino contribuyan a mejorar las estrategias generales de afrontamiento ante los problemas asociados con el consumo, las situaciones de riesgo, la abstinencia o las recaídas, tal y como sugieren Marlatt y Donovan (2005).

\section{Agradecimientos}

Este trabajo ha sido posible gracias a la ayuda a la actividad investigadora de la Fundación Proyecto Hombre de Navarra en colaboración con la Universidad Loyola Andalucía, el Servicio Navarro de Salud-Osasunbidea y la Orden Hospitalaria San Juan de Dios, año 2015.

\section{Conflicto de intereses}

Los autores declaran no tener conflictos de intereses.

\section{Referencias}

Alcoholics Anonymous. (2001). Alcoholics Anonymous (4th Ed.). New York, NY: A.A. World Services.

Andreasen, N.C. (1984). The Scale for the Assessment of Positive Symptoms (SAPS). Iowa City, IA: University of lowa.

Bachiller, D., Grau-López, L., Barral, C., Daigre, C., Alberich, C., Rodríguez-Cintas, L.,... Roncero, C. (2015). Grupo motivacional en unidad hospitalaria de desintoxicación, su influencia en mantenimiento de la abstinencia y retención al tratamiento tras alta. Adicciones, 27, 109-118.

Bacon, E. y Izaute, M. (2009). Metacognition in schizophrenia: Processes underlying patients' reflections on their own episodic memory. Biological Psychiatry, 66, 1031-1037. doi: 10.1016/j.biopsych.2009.07.013.

Bart, G. (2012). Maintenance medication for opiate addiction: The foundation of recovery. Journal of Addictive Diseases, 31, 207-225. doi:10.1080/10550887.2012.694598

Bateman, A.W. y Fonagy, P. (2006). Mentalization-Based Treatment for Borderline Personality Disorder: A Practical Guide. Oxford, UK: Oxford University Press. 
Caletti, E., Paoli, R.A., Florentini, A., Ciqqliobianco, M., Zugno, E., Serati, M. y Altamura, A.C. (2013). Neuropsychology, social cognition and global functioning among bipolar, schizophrenic patients and healthy controls: Preliminary data. Frontiers of Human Neuroscience, 7, 661. doi:10.3389/fnhum.2013.00661

Chambers, R.A. (2013). Adult hippocampal neurogenesis in the pathogenesis of addiction and dual diagnosis disorders. Drug and Alcohol Dependence, 130, 1-12. doi:10.1016/j.drugalcdep.2012.12.005

Chambers, R.A. y Potenza, M.N. (2003). Neurodevelopment, impulsivity, and adolescent gambling. Journal of Gambling Studies, 19, 53-84. doi:1050-5350/03/0300-0053/0

Chambers, R.A., Bickel, W.K. y Potenza, M.N. (2007). A scale-free systems theory of motivation and addiction. Neuroscience y Biobehavioral Reviews, 31, 1017-1045. doi:10.1016/j.neubiorev.2007.04.005

Chelton, L.G. y Bonney, L.C. (1987). Addiction, affects, and self-object theory. Psychotherapy: Theory, Research, Practice, Training, 24, 40-46. doi:10.1037/h0085689

De Rick, A., Vanheule, S. y Verhaeghe, P. (2009). Alcohol addiction and the attachment system: An empirical study of attachment style, alexithymia, and psychiatric disorders in alcoholic inpatients. Substance Use y Misuse, 44, 99-114. doi:10.1080/10826080802525744

Dinsmore, D.L., Alexander, P.A. y Loughlin, S.M. (2008). Focusing the conceptual lens on metacognition, self-regulation, and self-regulated learning. Educational Psychology Review, 20, 391-409.

Flavell, J.H. (1979). Metacognition and cognitive monitoring: A new area of cognitive-developmental inquiry. American Psychologist, 34, 906-911.

Greene, R.W., Biederman, J., Faraone, S.V., Wilens, T.E., Mick, E. y Blier, H.K. (1999). Further validation of social impairment as a predictor of substance use disorders: Findings from a sample of siblings of boys with and without ADHD. Journal of Clinical Child Psychology, 28, 349354. doi:10.1207/S15374424jccp280307

IBM Corp. Released 2012. IBM SPSS Statistics for Windows, Version 21.0. Armonk, NY: IBM Corp.

Ladegaard, N., Lysaker, P.H., Larsen, E.R. y Videbech, P. (2014). A comparison of capacities for social cognition and metacognition in first episode and prolonged depression. Psychiatry Research, 220, 883-889. doi:10.1207/ S15374424jccp280307

López-Duran, A., Becoña Iglesias, E., García Janeiro, J.M., Senra Comesaña, A., Cancelo Martínez, J.,... Díaz Castro, E. (2006) ¿Cómo evolucionan las personas con dependencia de la cocaína que están en tratamiento? Estudio a los tres y seis meses. Adicciones, 18, 327-336.

Luoma, J., Drake, C.E., Kohlenberg, B.S. y Hayes, S.C. (2011). Substance abuse and psychological flexibility: The development of a new measure. Addiction Research and Theory, 19, 3-13. doi:10.3109/16066359.2010.524956
Lysaker, P.H., Ringer, J.M., Buck, K.D., Grant, M.L.A., Olesek, K., Leudtke, B. y Dimaggio, G. (2012). Metacognitive and social cognition deficits in patients with significant psychiatric and medical adversity: A comparison between participants with schizophrenia and a sample of participants who are HIV-positive. Journal of Nervous y Mental Disease, 200, 130-134. doi: 10.1097/NMD. 0b013e3182439533

Lysaker, P.H. y Salyers, M.P. (2007). Anxiety symptoms in schizophrenia spectrum disorders: Associations with social function, positive and negative symptoms, hope and trauma history. Acta Psychiatrica Scandinavica, 116, 290-298. doi:10.1111/j.1600-0447.2007.01067.x

Lysaker, P.H., Bob, P., Pec, O., Hamm, J., Kukla, M., Vohs, J. y Dimaggio, G. (2013). Synthetic metacognition as a link between brain and behavior in schizophrenia. Translational Neuroscience, 4, 368-377. doi:10.2478/s13380-013-0131-4

Lysaker, P.H., Carcione, A., Dimaggio, G., Johannesen, J.K., Nicolò, G., Procacci, M. y Semerari, A. (2005). Metacognition amidst narratives of self and illness in schizophrenia: associations with insight, neurocognition, symptom and function. Acta Psychiatrica Scandinavica, 112, 64-71. doi:10.1111/j.1600-0447.2005.00514.x

Lysaker, P.H., Olesek, K., Buck, K.D., Leonhardt, B.L., Vohs, J., Ringer, J. y Outcalt, J. (2014). Metacognitive mastery moderates the relationship of alexithymia with cluster $\mathrm{C}$ personality disorder traits in adults with substance use disorders. Addictive Behaviors, 39, 558-561. doi:10.1016/j.addbeh.2013.11.007

Lysaker, P.H., Vohs, J., Minor, K.S., Irarrázaval, L., Leonhardt, B., Hamm, J.A.,... Dimaggio, G. (2015). Metacognitive Deficits in Schizophrenia: Presence and Associations with Psychosocial Outcomes. The Journal of Nervous and Mental Disease, 203, 530-536. doi:10.1097/ NMD.0000000000000323

Lyvers, M., Onuoha, R., Thorberg, F.A. y Samios, C. (2012). Alexithymia in relation to parental alcoholism, everyday frontal lobe functioning and alcohol consumption in a non-clinical sample. Addictive Behaviors, 37, 205-210. doi:10.1016/j.addbeh.2011.10.012

Marlatt, G.A. y Donovan, D.M. (2005). Relapse Prevention. Maintenance Strategies in the Treatment of Addictive Behaviors ( $2^{\text {nd }}$ Edition). Nueva York: Guildford Press.

McLeod, H.J., Gumley, A.I., MacBeth, A., Schwannauer, M. y Lysaker, P.H. (2014). Metacognitive functioning predicts positive and negative symptoms over 12 months in first episode psychosis. Journal of Psychiatry Research, 54, 109-115. doi:10.1016/j.jpsychires.2014.03.018

Muñiz, J., Elosua, P. y Hambleton, R.K. (2013). Directrices para la traducción y adaptación de los tests: segunda edición. Psicothema, 25, 151-157.

O'Brien, C.P. (2008). Evidence-based treatments of addiction. Philosophical Transactions of the Royal Society B: Biological Sciences, 363, 3277-3286. doi:10.1098/rstb.2008.0105 
OMS (1992). Décima revisión de la clasificación internacional de las enfermedades (CIE-10). Trastornos mentales y del comportamiento. Descripción clínica y pautas para el diagnóstico. Madrid: Editorial Méditor.

Ottavi, P., D’Alia, D., Lysaker, P.H., Kent, J., Popolo, R., Salvatore, G. y Dimaggio G. (2014). Metacognition-oriented social skills training for individuals with long-term schizophrenia: methodology and clinical illustration. Clinical Psychology y Psychotherapy, 21, 465-473. doi: 10.1002/cpp.1850

Pec, O., Bob, P. y Lysaker, P.H. (2015). Trauma, Dissociation and Synthetic Metacognition in Schizophrenia. Activitas Nervosa Superior, 57, 59-70.

Saladin, M.E., Santa Ana, E. J., LaRowe, S. D., Simpson, A. N., Tolliver, B. K., Price, K. L., ... Brady, K. T. (2012). Does alexithymia explain variation in cue-elicited craving reported by methamphetamine-dependent individuals? American Journal of Addiction, 21, 130-135. doi:10.1111/j.1521-0391.2011.00214.x

Sanz, J. y Navarro, M,E. (2003). Propiedades psicométricas de una versión española del Inventario de ansiedad de Beck (BAl) en estudiantes universitarios. Ansiedad y Estrés, 9, 59-84.

Semerari, A., Carcione, A., Dimaggio, G., Falcone, M., Nicolo, G., Procacci, M. y Alleva, G. (2003). How to evaluate metacognitive functioning in psychotherapy? The metacognition assessment scale and its applications. Clinical Psychology and Psychotherapy, 10, 238-261. doi:10.1002/cpp.362

Semerari, A., Colle, L., Pellecchia, G., Buccione, I., Carcione, A., Dimaggio, G.,... Pedone, R. (2014). Metacognitive dysfunctions in personality disorders: correlations with disorder severity and personality styles. Journal of Personality Disorders, 28, 751-766. doi:10.1521/ pedi_2014_28_137.

Semerari, A., Cucchi, M., Dimaggio, G., Cavadini, D., Carcione, A., Battelli, V., ... Smeraldi, E. (2012). The development of the Metacognition Assessment interview: instrument description, factor structure and reliability in a non-clinical sample. Psychiatry Research, 200, 890895. doi:10.1016/j.psychres.2012.07.015.

Tarricone, P. (2011). The taxonomy of metacognition. Ney York: Psychology Press.

Thorberg, F.A., Young, R.M., Sullivan, K.A. y Lyvers, M. (2009). Alexithymia and alcohol use disorders: A critical review. Addictive Behaviors, 34, 237-245. doi:10.1016/j. addbeh.2008.10.016

Thorberg, F.A., Young, R.M., Sullivan, K.A., Lyvers, M., Connor, J.P. y Feeney, G.F. (2011). Alexithymia, craving and attachment in a heavy drinking population. Addictive Behaviors, 36, 427-430. doi:10.1016.j.addbeh.2010.12.016

Toneatto, T. (1999). Metacognition and substance use. Addictive Behaviors, 24, 167-174. doi:10.1016/S03064603(98)00126-9 van Oosterhout, B., Smit, F., Krabbendam, L., Castelein, S., Staring, A.B. y van der Gaag, M. (2015). Metacognitive training for schizophrenia spectrum patients: a meta-analysis on outcome studies. Psychological Medicine, 20, 1-11. doi:10.1017/S0033291715001105

Verrocchio, M.C., Conti, C. y Fulcheri, M. (2010). Deliberate self-harm in substance-dependent patients and relationship with alexithymia and personality disorders: A case-control study. Journal of Biological Regulators and Homeostatic Agents, 24, 461-469.

Volkow, N.D., Fowler, J.S., Wang, G.J., Baler, R. y Telang, F. (2009). Imaging dopamine's role in drug abuse and addiction. Neuropharmacology, 56(s1), 3-9. doi:10.1016/j. neuropharm.2008.05.022

Wasmuth, S.L., Outcalt, J., Buck, K., Leonhardt, B.L., Vohs, J. y Lysaker, P.H. (2015). Metacognition in persons with substance abuse: Findings and implications for occupational therapists. Canadian Journal of Occupational Therapy, 82, 150-159. 